\title{
Dynamical Structure Factors of Quasi-One-Dimensional Magnets of the Type of $\mathrm{CsNiF}_{3}$
}

\author{
V. LisÝ* AND J. TÓTHOVÁ \\ Department of Physics, Faculty of Electrical Engineering and Informatics, Technical University of Košice \\ Park Komenského 2, 04200 Košice, Slovakia \\ By a direct derivation of the equations of motion for the spins in $\mathrm{CsNiF}_{3}$ we show that the extensively \\ used sine-Gordon equation fails to describe the dynamics of this $1 \mathrm{D}$ magnet. Instead of this soliton-bearing \\ model we use the spin-wave theory and, without going to the continuum approximation, calculate the dynamic \\ structure factors of the scattering of neutrons on $\mathrm{CsNiF}_{3}$. Complete analytical solutions for the dynamic structure \\ factors in the frequency domain are obtained both within the classical consideration and with quantum corrections.
}

PACS numbers: 75.10.Jm, 75.10.Hk, 75.30.Ds, 75.40.Gb, 78.70.Nx

\section{Introduction}

For more than three decades the magnetic compound $\mathrm{CsNiF}_{3}$ has attracted an intense attention of investigators [1]. This is mainly due to a relative simplicity of the Heisenberg model used to describe this magnet above the temperature $2.7 \mathrm{~K}$, where it exhibits essentially $1 \mathrm{D}$ behavior, and to a possible existence of nonlinear excitations of spins - integrable (solitonic) or non-integrable $[2,3]$, depending on whether the externally applied magnetic field is directed parallel or normal to the anisotropic axis. In the work by Mikeska [4], the fully integrable sine-Gordon (SG) model was proposed to describe the spin dynamics in $\mathrm{CsNiF}_{3}$. He has also calculated the "parallel" dynamic structure factor (DSF) of inelastic scattering of neutrons on kink and antikink solitons of the SG model. This and other results of the soliton theory were soon doubted by several authors [5], first in the work [6]. However, flaws of the derivation of the classical SG equation for $\mathrm{CsNiF}_{3}$ are, to our knowledge, not directly shown in the literature. In this contribution we thus returned to the original model and obtained equations of motion for the spins. We have found that the soliton theory is a very crude approximation for $\mathrm{CsNiF}_{3}$. Especially for large fields and low temperatures the spin-wave (SW) theory, without going to the continuum approximation, is much more substantiated. We have calculated, both in the classical limit and with quantum corrections, the longitudinal (with respect to the applied field oriented perpendicularly to the chain of spins) and transversal DSF of inelastic neutron scattering. As distinct from the previous calculation by Reiter [6], exact analytical expressions in the frequency domain have been obtained for the DSF which explicitly satisfies the detailed balance condition. The analysis shows that the DSF peaks - the central peak and the satellite ones - are always separated. The static structure factor has been also found. We thus propose the most complete solution for the DSF of 1D magnets of the type of $\mathrm{CsNiF}_{3}$ within the SW theory. The

* corresponding author; e-mail: vladimir.lisy@tuke.sk obtained formulae can be easily used for numerical calculations and the comparison with experiments.

\section{Failure of the SG model for $\mathrm{CsNiF}_{3}$}

In a number of investigations it is assumed that above the $3 \mathrm{D}$ ordering temperature the magnet $\mathrm{CsNiF}_{3}$ can be described by the spin $s=1$ Hamiltonian with a single-site anisotropy and a ferromagnetic exchange interaction,

$$
H=-\sum_{R R^{\prime}} J_{R R^{\prime}} \boldsymbol{S}_{R} \boldsymbol{S}_{R^{\prime}}+A \sum_{R}\left(S_{R}^{z}\right)^{2}-g \mu_{\mathrm{B}} B \sum_{R} S_{R}^{x},
$$

where the $z$ axis is along the chain and the Zeeman term is produced by an applied field $B$. Let us consider the system as being classical [4] and represent the spin vector as $\boldsymbol{S}=S\left\{\cos \theta_{R} \cos \phi_{R}, \cos \theta_{R} \sin \phi_{R}, \sin \theta_{R}\right\}$, where $\theta$ is the angle between the spin and its projection onto the plane $x y$ and $\phi$ is the angle between this projection and the axis $x$. The equations of motion for the spins, i $\hbar \partial S_{R}^{z}(t) / \partial t=\left[S_{R}^{z}, H\right]$, etc., for small $\theta$ and $\phi_{l}-\phi_{l+1}$ are then $\dot{\phi}_{l}=2(A S / \hbar) \theta_{l}+\left(g \mu_{\mathrm{B}} B \hbar\right) / \theta_{l} \cos \phi_{l}+$ $(J S / \hbar)\left(2 \theta_{l}-\theta_{l-1}-\theta_{l+1}\right)$ and $\dot{\theta}_{l}=-(J S / \hbar)\left(2 \phi_{l}-\phi_{l-1}-\right.$ $\left.\phi_{l+1}\right)-\left(g \mu_{\mathrm{B}} B / \hbar\right) \sin \phi_{l}$, with the constant $A$ changed to $A(S-1 / 2) / S[7]$. Only the nearest-neighbor interactions have been taken into account, $2 J_{R R^{\prime}}=J\left(\delta_{R^{\prime}, R+1}+\right.$ $\left.\delta_{R^{\prime}, R-1}\right)$. For low fields $\left(g \mu_{\mathrm{B}} B \ll 2 A S\right)$ and neglecting the last term in the second of these equations, we come in the continuum approximation to the SG model [4], $\phi_{z z}-c^{-2} \phi_{t t}=m^{2} \sin \phi$, where $c^{2} \equiv 2 A J S^{2} a^{2} / \hbar^{2}$, $m^{2} \equiv g \mu_{\mathrm{B}} B /\left(J S a^{2}\right)$, and $a$ is the lattice constant. However, it is easy to see that this approximation is very crude. First, the continuum approximation that requires $m a \ll 1$ does not hold well for usual experimental fields and standard parameters $(A=4.5 \mathrm{~K}, J=23.6 \mathrm{~K}$, $g=2.4$ ). So, for $B=5 \mathrm{kG}, m a$ is about 0.2 and increases with the field. On the other hand, for lower fields one cannot assume small $\theta$. In the Fourier transformation (FT) $\theta_{n}=N^{-1 / 2} \sum_{k} \exp (-\mathrm{i} k n) \theta_{k}(k=2 \pi n / N a$, $n=1,2, \ldots, N / 2)$ one finds that the required condition $A \gg J(1-\cos k a)$ is poorly satisfied for the experimental wave vectors (e.g., if $a k=0.1 \pi$, only as $4 \gg 1$, for larger $k$ even worse; at $0.2 \pi$ both terms are about equal). The best satisfied condition $g \mu_{\mathrm{B}} B \ll 2 A S$ holds for $B=5 \mathrm{kG}$ 
only as $1 \ll 10$. Overall, it is seen that the SG model from which the soliton theory follows is not appropriate for $\mathrm{CsNiF}_{3}$ at all.

\section{Spin-wave approach and the dynamic structure factors}

Especially at low temperatures and large fields, without going to the continuum approximation, the SW theory suits better for the description of the $\mathrm{CsNiF}_{3}$ dynamics. The spins preferably orient along the field $\boldsymbol{B}$, which allows us to linearize the problem using $\sin \phi \approx \phi$ and $\cos \phi \approx 1$. Then in the FT we have $\hbar S \dot{\theta}_{k}=-M_{k} a^{2} \omega_{k}^{2} \phi_{k}$ and $a^{2} M_{k} \dot{\phi}_{k}=\hbar S \theta_{k}$. The frequency and effective mass of magnons are $\omega_{k}^{2}=(2 J S / \hbar)^{2}(P-\cos k a)(Q-\cos k a)$ and $M_{k}=\left(\hbar^{2} / 2 J a^{2}\right)(Q-\cos k a)^{-1}$, where $P=1+$ $g \mu_{\mathrm{B}} B / 2 J S$ and $Q=P+A / J$. For the canonical momenta $p_{i k}$ and coordinates $x_{i k}\left(i=1,2, p_{1 k}+\mathrm{i} p_{2 k}=\right.$ $\left.\sqrt{2} \hbar S \theta_{k} / a, x_{1 k}+\mathrm{i} x_{2 k}=\sqrt{2} a \phi_{k}\right)$ the Hamiltonian takes a diagonal form. It allows us to easily find the mean values and correlation functions of the generalized variables, and consequently the DSF. We have completely solved this problem and found exact analytical expressions for the structure factors $\left\langle S_{q}^{\alpha}(t) S_{-q}^{\alpha}\right\rangle$ and their FTs, which determine the DSF $S^{\alpha \alpha}(q, \omega)$ for 1D magnets $(\alpha=x, y$, $z$ and $q$ is the $z$ component of the scattering vector).

We have found that $S^{y y}+S^{z z}$ contains peaks at the frequencies $\omega= \pm \omega_{q}$ and a weak central peak (CP), and in $S^{x x}$ there are both the $\mathrm{CP}$ and the satellite peaks, $S^{x x}(q, \omega)=S_{\mathrm{C}}(q, \omega)+S_{\text {sat }}(q, \omega)$, which are always separated. The results qualitatively correspond to the experiments [8], however, the condition $\hbar \omega_{q} / k_{\mathrm{B}} T \ll 1$ is not satisfied. The quantum corrections are thus necessary.

To find them, $H$ can be expressed in the form $H=$ $\hbar \sum_{k} \omega_{k} b_{k}^{+} b_{k}$, where $\left[b_{k}, b_{k^{\prime}}^{+}\right]=\delta_{k k^{\prime}},\left[b_{k}, b_{k^{\prime}}\right]=0$, $\left\langle b_{k}^{+} b_{k}\right\rangle=n_{k}=\left[\exp \left(\hbar \omega_{k} / k_{\mathrm{B}} T\right)-1\right]^{-1}$, and $\left\langle b_{k} b_{k}^{+}\right\rangle=$ $n_{k}+1$. The transfer from the classical Hamiltonian to that of a Bose gas is realized using the following relations between the operators: $\hat{x}_{k}=\sqrt{\hbar /\left(2 M_{k} \omega_{k}\right)}\left(b_{k}+b_{-k}^{+}\right)$ and $\hat{p}_{k}=\mathrm{i} \sqrt{M_{k} \hbar \omega_{k} / 2}\left(b_{k}^{+}-b_{-k}\right)$. The time dependence of the operators is given by $b_{k}(t)=b_{k} \exp (-\mathrm{i} \omega t)$. By this way one easily finds all the DSFs, which for $n_{q} \approx k_{\mathrm{B}} T / \hbar \omega_{q} \gg 1$ coincide with the classical results. Here we give only the final formula for $S^{x x}(q, \omega)$ when $q>0, \omega \geq 0$ ( $\omega=0$ gives the static structure factor):

$$
\begin{aligned}
& S_{\mathrm{C}}(q, \omega)=\frac{2}{\pi}\left(\frac{\hbar}{4 J S}\right)^{3} \sum_{i}\left[\omega_{k} \omega_{q-k}(F+G)\right. \\
& \left.\quad \times n_{q-k}\left(n_{k}+1\right)\right]_{k=k_{i}}\left|f_{+}\left(k_{i}\right)\right|^{-1}, \\
& S_{\text {sat }}(q, \omega)=\frac{1}{\pi}\left(\frac{\hbar}{4 J S}\right)^{3} \sum_{i}\left[\omega_{k} \omega_{q-k}(F-G)\right. \\
& \left.\quad \times\left(n_{q-k}+1\right)\left(n_{k}+1\right)\right]_{k=k_{i}}\left|f_{-}\left(k_{i}\right)\right|^{-1},
\end{aligned}
$$

where $k_{i}\left(\left|k_{i}\right|<\pi / a\right)$ are the solutions of the equation $\omega-\omega_{k}+\omega_{q-k}=0$ for $S_{\mathrm{C}}$ and $\omega-\omega_{k}-\omega_{q-k}=0$ for $S_{\text {sat }}$. The functions $F$ and $G$ are defined by the relation $F \pm G=\left\{[(P-\cos k a)(P-\cos (k a-q a))]^{-1 / 2} \pm(P \rightarrow Q)\right\}^{2}$ and $f_{ \pm}$are determined as

$$
\begin{gathered}
f_{ \pm}(k)= \pm \sin (k a-q a)\left[\frac{P-\cos (k a-q a)}{Q-\cos (k a-q a)}\right]^{1 / 2} \\
-\sin k a\left[\frac{P-\cos k a}{Q-\cos k a}\right]^{1 / 2}+(P \Leftrightarrow Q) .
\end{gathered}
$$

As distinct from the result in [6], $S^{x x}(q, \omega)$ automatically obeys the detailed balance condition $S^{x x}(q,-\omega)=$ $\exp \left(-\hbar \omega / k_{\mathrm{B}} T\right) S^{x x}(q, \omega)$.

\section{Conclusions}

Our interest to $\mathrm{CsNiF}_{3}$ was stimulated by the frequent assumption in the literature that the dynamics of this 1D magnet can be described by the SG model possessing soliton solutions. In the present paper we have re-derived the classical equations of motion for the spins and showed that this model is not appropriate for the description of the $\mathrm{CsNiF}_{3}$ dynamics. Much more suitable, especially for large external fields, is the model of magnon excitations. We have obtained complete analytical solutions for the DSFs of inelastic neutron scattering on $\mathrm{CsNiF}_{3}$ both in the classical consideration and with quantum corrections, in the case when the field is perpendicular to the chain of spins. The found solutions are suitable for numerical calculations since they require only finding roots of simple equations for the scattering wave vectors.

It should be however noted that this work is rather of a methodical interest and does not pretend to an exact description of experiments. In fact, the comparison with the neutron scattering experiments shows notable departures from the theory, particularly at low temperatures when the SW model should work well. However, by lowering the temperature down to the 3D-ordering temperature it is expected that the adequacy of the simple 1D model decreases. On the other hand, at higher temperatures the model could be good but the SW approximation becomes less appropriate. We conclude that despite the apparent simplicity of the studied system and great interest to it an acceptable theory of its dynamics is only to be created.

\section{Acknowledgments}

This work was supported by the Agency for the Structural Funds of the EU within the project NFP 26220120021 , and by the grant VEGA $1 / 0300 / 09$.

\section{References}

[1] M. Orendáč, A. Orendáčová, E. Čižmár, J.-H. Park, A. Feher, S.J. Gamble, S. Gabáni, K. Flachbart, J. Karadamoglou, M. Poirier, M.W. Meisel, Phys. Rev. B 69, 184403 (2004).

[2] J.P. Nguenang, T.C. Kofane, Chaos Solitons Fractals 30, 552 (2006).

[3] J. Beula, M. Daniel, Physica D 239, 397 (2010).

[4] H.J. Mikeska, J. Phys. C, Solid State Phys. 11, L29 (1978).

[5] V.G. Makhankov, Soliton Phenomenology, Kluwer, Dordrecht 1992.

[6] G. Reiter, Phys. Rev. Lett. 46, 202 (1981).

[7] J. Karadamoglou, N. Papanicolaou, Phys. Rev. B 63, 224406 (2001).

[8] M. Steiner, K. Kakurai, W. Knop, R. Pynn, J.K. Kjems, Solid State Commun. 41, 329 (1982). 